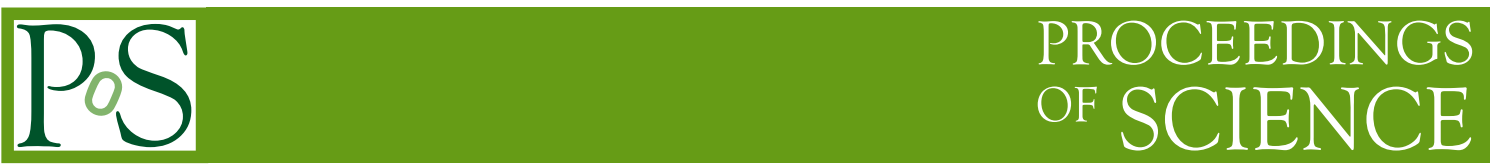

\title{
Triggering in the ATLAS experiment
}

\author{
Javier Montejo Berlingen, on behalf of the ATLAS Collaboration ${ }^{a, *}$ \\ ${ }^{a}$ CERN, Geneva, Switzerland \\ E-mail: jmontejo@cern.ch
}

The ATLAS experiment at the LHC can record about $1 \mathrm{kHz}$ of physics collisions, out of an LHC design bunch crossing rate of $40 \mathrm{MHz}$. To achieve a high selection efficiency for rare physics events (such as beyond the Standard Model physics) while reducing the large background rate, a two-level trigger system is used. The event selection is based on physics signatures, such as the presence of energetic leptons, photons, jets or missing energy. In addition, the trigger system exploits algorithms using topological information and multivariate methods to carry out the filtering for the many physics analyses pursued by the ATLAS collaboration. In Run 2, around 1500 individual selections, the trigger chains, are included in the trigger menu specifying the selection algorithms to be used for data taking. Trigger menus must reflect the physics goals for a given data-taking period, taking the instantaneous luminosity of the LHC and limitations from the ATLAS detector readout and offline processing farm into account. An overview of the 2015-2018 trigger system and its performance is presented.

40th International Conference on High Energy physics - ICHEP2020

July 28 - August 6, 2020

Prague, Czech Republic (virtual meeting)

\footnotetext{
${ }^{*}$ Speaker
} 


\section{Introduction}

The ATLAS detector is a multipurpose particle physics detector with nearly $4 \pi$ coverage in solid angle around the collision point, and is described in detail in Ref. [1]. The trigger system [2] is responsible for the selection of collisions to be recorded and analysed offline. It has operated successfully during the second run of LHC [3], from 2015 to 2018, where the LHC delivered peak luminosities exceeding $L=2.0 \times 10^{34} \mathrm{~cm}^{-2} \mathrm{~s}^{-1}$.

In Run 2 the ATLAS detector had a two-level trigger system. The first-level trigger (Level-1 trigger, L1) is implemented in custom hardware and uses a subset of the detector information to reduce the rate of accepted events from an input rate of up to $40 \mathrm{MHz}$ to maximally $100 \mathrm{kHz}$. It consists of the L1Calo and the L1Muon subsystems, which process the inputs from the calorimeter and the muon detectors, respectively. In addition, the L1 topological trigger (L1Topo) performs topology-based selections using inputs from the calorimeter and muon systems. The most common among such selections are cuts on the angular distance, or on the invariant mass of pairs of particle candidates. After the L1 decision, the full detector data are readout and temporarily buffered, and the second level trigger decides whether the event should be saved to tape. The second level of the trigger system is a software-based trigger (high-level trigger, HLT) that reduces the rate of recorded events to $1 \mathrm{kHz}$ on average. The event reconstruction at the HLT of objects such as leptons or jets happens only to the extent required by the executed trigger algorithms.

During Run 2, the ATLAS trigger system was continuously optimised and improved in order to cope with increasingly higher event rates and larger number of simultaneous proton-proton interactions (pile-up). Some representative examples of such improvements are described here.

L1 calorimeter trigger: new filter coefficients and noise cuts were optimised for the high pile-up conditions reached in the latter half of Run 2. This significantly reduced the rates of triggers that are most sensitive to pile-up, such as missing transverse energy $\left(E_{\mathrm{T}}^{\mathrm{miss}}\right)$ and low- $p_{\mathrm{T}}$ multijet triggers, and also reduced the non-linear dependence of these rates on the pile-up. The reduced calorimeter occupancy after the noise cuts also improved the reconstruction time at the HLT level.

L1 muon trigger: a coincidence with the inner muon chamber located before the toroid magnet $(1.05<|\eta|<2.0)$ was used to reduce the rate of fake muon candidates. An additional coincidence with an energy deposition in the hadronic calorimeter was introduced in the $\eta$ region with poor coverage of the inner muon chamber $(1.05<|\eta|<1.3)$. The coincidences reduced the rate by about $30 \%$ for the primary L1 muon trigger, with an efficiency loss below $1 \%$.

HLT: the constant improvement of identification algorithms and pile-up rejection methods allowed to keep almost constant kinematic thresholds throughout Run 2, despite the increase in luminosity. Further details of the algorithms and improvements are discussed in Section 3.

\section{Trigger menu}

The set of event selections at L1 and HLT that lead to the decision of storing or rejecting an event are referred to simply as triggers, and the collection of all triggers is called the trigger menu. 
The Run-2 trigger menu was designed to record and provide data for analyses, maintaining the same online $p_{\mathrm{T}}$ requirements, also referred to as thresholds, across the different years. This enabled the trigger system to provide a consistent dataset despite the higher luminosity and pile-up during the last two years of Run 2 with respect to the first two. An exhaustive list of triggers included in the Run-2 ATLAS trigger menu is documented in Ref [4].

The design of the trigger menu is based on the following building blocks:

- Primary triggers, which are used for physics measurements.

- Support triggers, which are used for efficiency and performance measurements, background estimates or monitoring, and typically run at a small rate (of the order of $0.5 \mathrm{~Hz}$ each). About $15 \%$ of the HLT bandwidth is dedicated to support triggers.

- Alternative triggers, which run non-standard online reconstruction algorithms complementary to primary and/or support triggers. For example as part of the commissioning of future primaries.

- Backup triggers, with tighter selections and lower expected rate, in case the rate of the primary trigger becomes too high.

- Calibration triggers, which are used for detector calibrations and often run at high rate but store very small-size events with only the relevant information from the detector to be calibrated.

\section{Trigger performance}

Most of the trigger reconstruction and identification algorithms were improved during Run 2 in order to better cope with the challenging pile-up conditions. A non-exhaustive list is described in the following, providing some representative examples. A complete list of improvements can be found in Refs. [4-6].

\subsection{Missing energy trigger}

As $E_{\mathrm{T}}^{\text {miss }}$ is a global event-level variable, the rate of $E_{\mathrm{T}}^{\text {miss }}$ triggers is strongly affected by the number of collisions per bunch crossing, since each proton-proton collision adds to the total energy deposited in the calorimeter. While complex correction techniques mitigate these effects to a large extent in the offline reconstruction, at trigger level these effects lead to a strong increase of the trigger rate with increasing pile-up. As such, constant improvements were introduced during Run 2 in order to run efficiently at high luminosity [7].

Several algorithms were developed in order to reconstruct the $E_{\mathrm{T}}^{\text {miss }}$ at the HLT. The cell $E_{\mathrm{T}}^{\text {miss }}$ is reconstructed considering all calorimeter cells above a certain noise threshold. The missing $H_{\mathrm{T}}(m h t)$ algorithm calculates $E_{\mathrm{T}}^{\mathrm{miss}}$ as the negative vector sum of transverse momentum of calibrated anti$k_{t}$ [8] jets with radius parameter $\mathrm{R}=0.4$, where those jets are constructed from calibrated topological clusters made of calorimeter cells. The pufit algorithm is designed to disentangle calorimeter deposits from the hardest proton-proton interaction (hard-scatter), from those originating from pileup interactions. Towers made out of topological clusters are grouped into pile-up and hard-scatter categories based on their energy. The energy threshold to separate into both categories is dependent 
on the mean energy and the variance of low- $p_{\mathrm{T}}$ towers. Assuming that the contribution to $E_{\mathrm{T}}^{\text {miss }}$ from pile-up interactions averages to be zero, a minimisation taking into account resolution terms determines energy contributions from pile-up interaction in the hard-scatter towers. These pile-up contributions are then subtracted, and the final $E_{\mathrm{T}}^{\text {miss }}$ value is determined from the negative sum of transverse energy of those pile-up corrected hard-scatter towers. The default $E_{\mathrm{T}}^{\text {miss }}$ reconstruction algorithm has evolved during Run 2 towards more pileup-robust algorithms, from cell, to $m h t$, to pufit as the peak luminosity increased. During 2018 a combination of selections on pufit and cell $E_{\mathrm{T}}^{\text {miss }}$ was used. This combination yielded a significantly reduced rate while retaining the same efficiency with respect to the offline $E_{\mathrm{T}}^{\text {miss }}$ threshold. The trigger efficiency as a function of the offline $E_{\mathrm{T}}^{\mathrm{miss}}$ threshold for each of the triggers is shown in Figure 1.

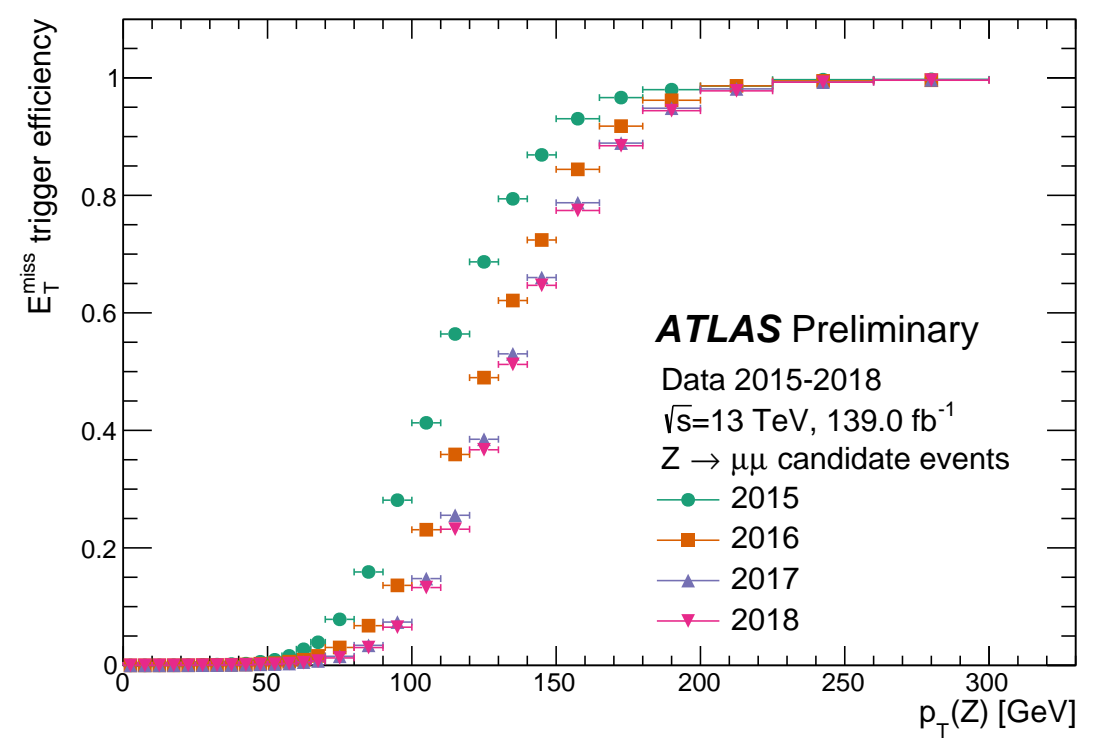

Figure 1: The combined L1 and HLT efficiency of the lowest unprescaled missing transverse energy triggers for the years 2015 to 2018 are shown as a function of the $Z$ boson transverse momentum [9]. The events are taken from data with a $Z \rightarrow \mu \mu$ selection, and the transverse momentum of the $\mathrm{Z}$ boson is used as a proxy for the missing transverse momentum in the event, as muons are treated as invisible objects by the triggers concerned. Depending on the data-taking period, the HLT $E_{\mathrm{T}}^{\text {miss }}$ was calculated with the "cell", "mht", "pufit" or "cell+pufit" algorithm, during 2015, 2016, 2017 and 2018 respectively. The peak luminosity and pile-up differ among the years.

\subsection{Tau trigger}

Tau leptons are a key signature for many searches and measurements of Standard Model processes. Tau leptons decay either leptonically $\left(\tau \rightarrow \ell v_{\ell} v_{\tau}, \ell=e, \mu\right)$ or hadronically $(\tau \rightarrow$ hadrons $v_{\tau}$ ). The hadronic decays represent $65 \%$ of all possible tau decay modes, and triggering on the hadronic tau decays is critical for the sensitivity of analyses.

Dedicated tau trigger algorithms were designed and implemented based on the main features of hadronic tau decays: narrow calorimeter energy deposits and a small number of associated tracks [10]. The triggers run the same algorithms as used offline, in some cases with small 
variations. Due to the high production rate of jets with features similar to hadronic tau decays, keeping the rate of tau triggers under control is challenging.

An improved tau trigger was introduced in 2018. It implements a recurrent neural network (RNN) for improved identification [11], and Boosted Regression Tree for energy calibration. It also adds a new 0 -track category in order to recover track finding inefficiencies during the first tracking step. The higher background rejection power allowed to improve the efficiency while keeping the same rate. The improvement is especially visible in the high- $p_{\mathrm{T}}$ regime, as shown in Figure 2.

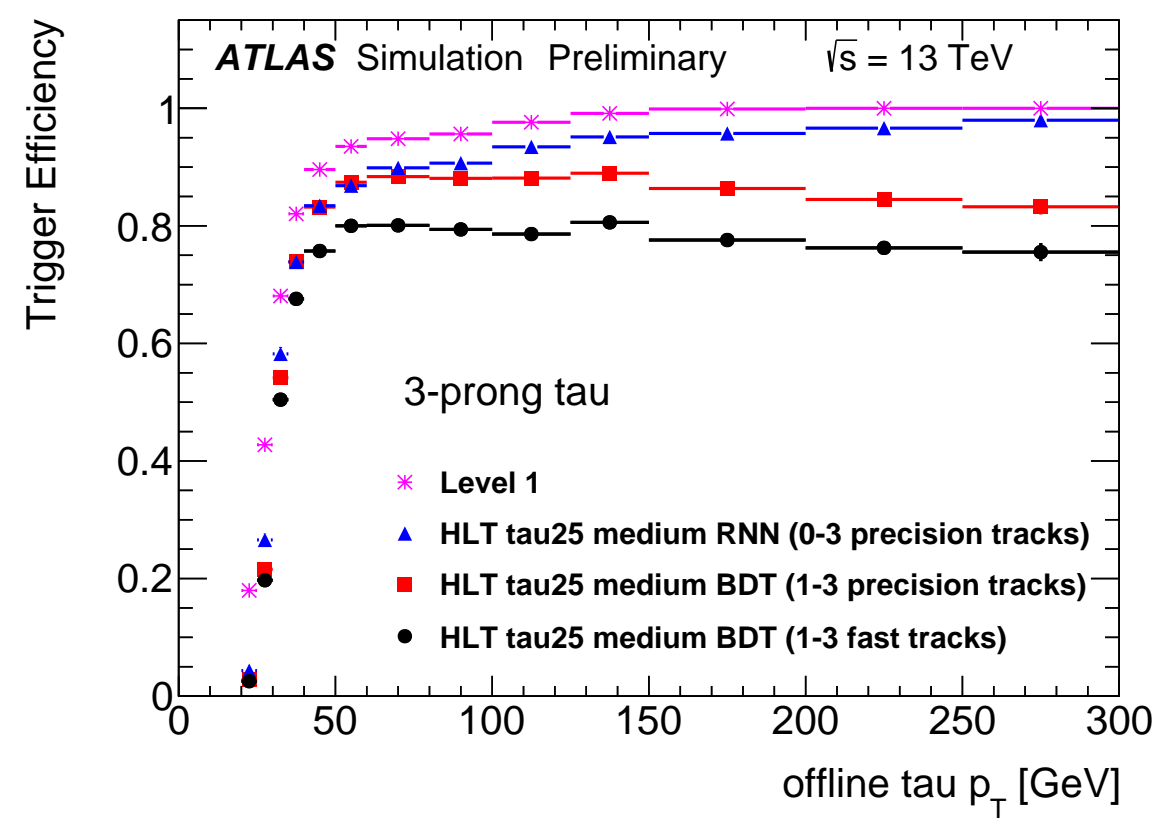

Figure 2: Efficiency of $\tau$-lepton triggers as a function of the offline transverse momentum, for an $E_{\mathrm{T}}$ threshold of $12 \mathrm{GeV}$ and a medium isolation requirement at Level 1, and an $E_{\mathrm{T}}$ threshold of $25 \mathrm{GeV}$ at the High Level Trigger (HLT) [12]. The triggers with boosted decision tree (BDT) identification and fast tracks selection, BDT identification and precision tracks selection, and RNN identification with precision tracks selection were deployed in 2016, 2017 and 2018, respectively. The three HLT versions have comparable trigger rates.

\section{3 $B \rightarrow K^{*} e e$ trigger}

In view of the intriguing results on lepton flavour violation in $R_{K}$ and $R_{K}^{*}$ measurements [13, 14], dedicated triggers were introduced in 2018, targeting both resolved and merged dielectron final states, as illustrated in Figure 3. Several new triggers were introduced with different strategies.

Resolved L1: require two separated electrons with $E_{\mathrm{T}}>7 / 5 \mathrm{GeV}$ and a dielectron mass below $9 \mathrm{GeV}$. In order to further reduce the rate, additionally one muon with $p_{\mathrm{T}}>6 \mathrm{GeV}$ or two muons with $p_{\mathrm{T}}>4 \mathrm{GeV}$ are required. The additional muon selection exploits the correlated production of B-hadron pairs in order to increase the purity with a muon on the opposite B-hadron.

Merged L1: require one electron with $E_{\mathrm{T}}>7 \mathrm{GeV}$ close to a jet with $E_{\mathrm{T}}>15 \mathrm{GeV}$. The small angular separation between the two electrons prevents them from being separately 
reconstructed. However the sum of both is reconstructed as a jet. The same muon selection as in the resolved trigger is also applied.

Unseeded L1: the HLT algorithm is run on every L1-accepted event. No additional requirement is placed at $\mathrm{L} 1$.

HLT : the trigger selects two $5 \mathrm{GeV}$ electrons, originating from a common vertex and with an invariant mass $\left(m_{e e}\right)$ of the two candidates within $0.1 \mathrm{GeV}<m_{e e}<6 \mathrm{GeV}$. When the algorithm is run over the merged or resolved L1 triggers a similar requirement for muons at HLT is placed. No muon is required when running in the unseeded mode.

The operation of this set of HLT triggers is computationally intensive, as it requires to run low$p_{\mathrm{T}}$ electron reconstruction on every electron L1 candidate down to the lowest possible threshold. The triggers had to be prescaled at peak luminosity in order to keep the rate below $120 \mathrm{~Hz}$, and run unprescaled below $1.85 \times 10^{34} \mathrm{~cm}^{-2} \mathrm{~s}^{-1}$.
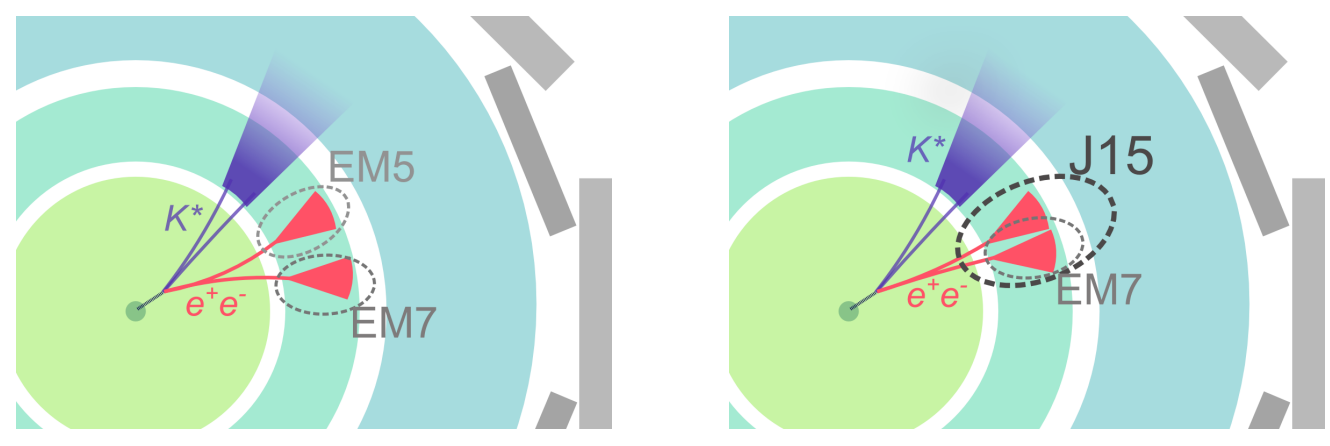

Figure 3: Sketch of the resolved (left) and merged (right) decay topologies. For decays with small angular distance the reconstruction of two separate electrons candidates at L1 is not possible, and triggers for the merged topology are used [4].

\section{Conclusions}

The ATLAS trigger system was successfully operated during the LHC Run-2 data-taking period. The trigger menu allowed for the collection of a balanced data set for various physics analyses as well as for detector monitoring and calibration purposes. The improvements and developments to the trigger system and its algorithms, with the focus on high pile-up scenarios, have allowed ATLAS to record data efficiently during Run 2 and cope with the significant increase in instantaneous luminosity. 


\section{References}

[1] ATLAS Collaboration. The ATLAS Experiment at the CERN Large Hadron Collider. JINST, 3:S08003, 2008.

[2] ATLAS Collaboration. Performance of the ATLAS Trigger System in 2015. Eur. Phys. J. C, 77(5):317, 2017.

[3] ATLAS Collaboration. Operation of the ATLAS trigger system in Run 2. JINST, 15(10): P10004, 2020.

[4] ATLAS Collaboration. Trigger menu in 2018, ATL-DAQ-PUB-2019-001. URL https: //cds.cern.ch/record/2693402.

[5] ATLAS Collaboration. Trigger Menu in 2017, ATL-DAQ-PUB-2018-002. URL https: //cds. cern.ch/record/2625986.

[6] ATLAS Collaboration. Trigger Menu in 2016, ATL-DAQ-PUB-2017-001. URL https: //cds. cern.ch/record/2242069.

[7] ATLAS Collaboration. Performance of the missing transverse momentum triggers for the ATLAS detector during Run-2 data taking. JHEP, 08:080, 2020.

[8] Matteo Cacciari, Gavin P. Salam, and Gregory Soyez. The anti- $k_{t}$ jet clustering algorithm. JHEP, 04:063, 2008.

[9] ATLAS Collaboration. ATLAS Missing energy trigger public results, 2018. URL https:// twiki.cern.ch/twiki/bin/view/AtlasPublic/MissingEtTriggerPublicResults.

[10] ATLAS Collaboration. The ATLAS Tau Trigger in Run 2, ATLAS-CONF-2017-061. URL https://cds. cern. ch/record/2274201.

[11] ATLAS Collaboration. Identification of hadronic tau lepton decays using neural networks in the ATLAS experiment, ATL-PHYS-PUB-2019-033. URL http://cds . cern . ch/record/ 2688062.

[12] ATLAS Collaboration. ATLAS Tau trigger public results, 2018. URL https://twiki. cern.ch/twiki/bin/view/AtlasPublic/TauTriggerPublicResults.

[13] LHCb Collaboration. Search for lepton-universality violation in $B^{+} \rightarrow K^{+} \ell^{+} \ell^{-}$decays. Phys. Rev. Lett., 122:191801, 2019.

[14] LHCb Collaboration. Test of lepton universality with $B^{0} \rightarrow K^{* 0} \ell^{+} \ell^{-}$decays. JHEP, 08:055, 2017. 